\title{
Manipulating Molecular Weight Distributions via "Locked-Unlocked" Anionic Polymerization
}

Cun Li ${ }^{l}$, Li Han ${ }^{l}$, Hongyuan Bai ${ }^{1}$, Xuefei Wang ${ }^{l}$, Yu Yin ${ }^{1}$, Hong Yan ${ }^{l}$, Xiaolu Zhang $^{1}$, Zheng Yang ${ }^{1}$, Pibo Liu ${ }^{* 2}$, Hongwei Ma*1

1. Department of Polymer Science and Engineering, School of Chemical Engineering, Dalian University of Technology, Dalian 116024, China

2. Division of Energy Materials, Dalian Institute of Chemical Physics, Chinese Academy of Sciences, Dalian 116023, China

*Email:mahw@dlut.edu.cn (M. H. W.); pibo.liu@dicp.ac.cn (L. P. B.)

Table of the Content

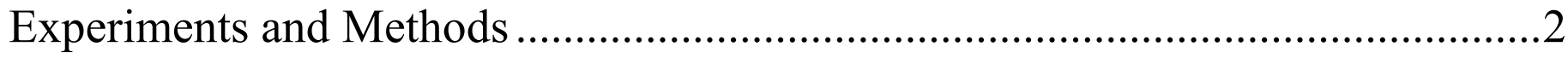

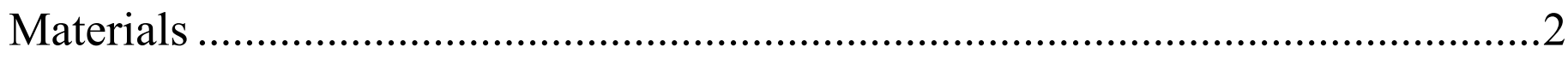

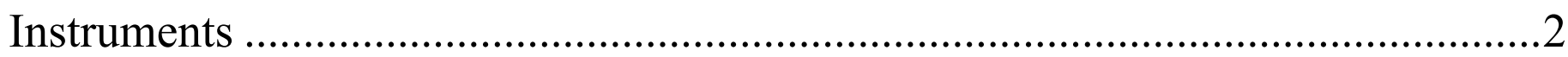

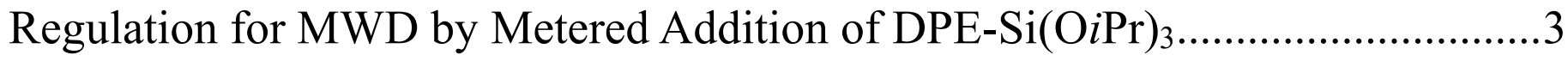

Synthesis of the Block-Graft Copolymer N/B/MP-PS- $b$-PSD-g-(PIp) $)_{n} \ldots \ldots \ldots \ldots \ldots . . . . . .3$

Additional Characteristics of the Polymers............................................................... 


\section{Experiments and Methods}

\section{Materials}

Tetraisopropylorthosilicate (Energy Chemical, 98.0\%), 4-bromobenzophenone (Energy Chemical, 98.0\%), methyltriphenylphosphonium bromide (Energy Chemical, 98.0\%), potassium tert-butoxide (Energy Chemical, 98.0\%), magnesium turnings for the Grignard reaction (STREM, 99\%). Tetrahydrofuran (THF) was dried by refluxing over a Na-benzophenone complex. Benzene (Certified ACS, EM Science), isoprene (Aladdin, 99.0\%) and styrene (Aldrich, 99\%) were purified as previously described ${ }^{1}$. The concentration of $s$-BuLi was 0.30 mol $\mathrm{L}^{-1} . n$-butyllithium (Macklin, $1.60 \mathrm{~mol} \mathrm{~L}^{-1}$ ) was used as purchased. The concentration of potassium tertbutoxide $\left(t\right.$-BuOK) was $0.20 \mathrm{~mol} \mathrm{~L}^{-1}$. 1-(4-triisopropoxysilylphenyl)-1-phenylethylene (DPE-Si(OiPr) 3 , 1-(4dimethylsilylphenyl)-1-phenylethylene (DPE-SiH) and trimethyl((4-(1-phenylvinyl)-phenyl)ethynyl) silane (DPE-yne) were all synthesized as the publications ${ }^{2-4}$.

\section{Instruments}

${ }^{1} \mathrm{H}-\mathrm{NMR}\left(5 \mathrm{wt} \%, \mathrm{CDCl}_{3}\right)$ spectra were recorded with a Bruker Advance II $400 \mathrm{MHz}$ NMR spectrometer with $\left(\mathrm{CH}_{3}\right)_{4} \mathrm{Si}$ (tetramethylsilane, TMS) as an internal standard. Size exclusion chromatographic (SEC) analyses of the polymers were performed with a Waters HPLC component system (2414 refractive index detector) at a flow rate of $1.00 \mathrm{~mL} \mathrm{~min}^{-1}$ in $\mathrm{THF}$ at $30{ }^{\circ} \mathrm{C}$ after calibration using polystyrene standard polymers. MALDI-TOF-MS analysis was carried out with a Waters MALDI micro-MX mass spectrometer. 2-[(2E)-3(4-tert-butylphenyl)-2-methyprop-2-enylidene] malonitrile (DCTB) and sodium trifluoroacetate were used as dopants; the details of the sample preparation are provided in a previous study ${ }^{5}$. The temperature sweep experiments (storage modulus and damping factor $(\tan \delta$ ) as functions of the scanning temperature) were performed by dynamic mechanical analysis (Q800, TA instrument Co., USA). The strain amplitude and frequency were fixed at $0.1 \%$ and $1 \mathrm{~Hz}$, respectively. The temperature was varied from -60 to $120{ }^{\circ} \mathrm{C}$, and the temperature rise rate was $3{ }^{\circ} \mathrm{C} \min ^{-1}$. The glass transition temperatures $(\mathrm{Tg})$ of the polymers were measured under a nitrogen atmosphere using a TA Instruments Universal Analysis 2000 differential scanning calorimeter 
(DSC) at temperatures varying from -100 to $120^{\circ} \mathrm{C}$ at a heating rate of $5.0{ }^{\circ} \mathrm{C} \mathrm{min}^{-1}$.

\section{Regulation for MWD by Metered Addition of DPE-Si(OiPr)3}

The different regulation entry of MWD using different additional protocol of DPE-Si $(\mathrm{O} i \mathrm{Pr})_{3}$ are similar. Thereby, the modulating sample named BP 1 shown in Table 1 was taken as an example for explaining the regulation process of MWD in LAP by the end-capping strategy. All the regulation experiments were operated under glovebox condition. Before the polymerization, quantitative DPE-Si(OiPr $)_{3}$ was diluted for late use. Benzene $(10 \mathrm{w} / \mathrm{v} \%)$ and styrene $(0.5 \mathrm{~g}, 4.8 \mathrm{mmol})$ were firstly added to a $20 \mathrm{~mL}$ sealed vial, and sec-BuLi $(83.0 \mu \mathrm{L}, 0.025 \mathrm{mmol})$ was injected to initiate styrene. After 20 minutes, a portion of DPE-Si(OiPr) $3(2.4 \mathrm{mg}$, $0.006 \mathrm{mmol}$ ) was injected to the system for locking part living species. And then this operation will be repeated one time every 20 minutes in the following 60 minutes. Finally, the polymer chains were totally end-capped four time in 80 minutes. After terminated with isopropanol, the product was precipitated with sufficient methanol and then dried in a vacuum oven to constant weight for further characterized.

\section{Synthesis of the Block-Graft Copolymer N/B/MP-PS-b-PSD-g-(PIp)n}

The preparations for three types block-graft copolymer N/B/MP-PS- $b$-PSD- $g$-(PIp) $)_{\mathrm{n}}$ are similar. Therefore, the synthesis of BP-PS- $b$-PSD- $g-(\mathrm{PIp})_{\mathrm{n}}$ was taken as an example here for explaining the operations. Before the polymerization, quantitative $\mathrm{DPE}-\mathrm{Si}(\mathrm{O} i \mathrm{Pr})_{3}$ was also diluted for late use. Then benzene $\left(10 \mathrm{w} / \mathrm{v}^{0} \mathrm{)}\right)$ and styrene $(2.00 \mathrm{~g}, 19.21 \mathrm{mmol})$ were firstly added to a $100 \mathrm{~mL}$ sealed vial, and $\mathrm{sec}$-BuLi $(333 \mu \mathrm{L}, 0.10 \mathrm{mmol})$ was injected to initiate styrene. After 15 minutes, a portion of DPE-Si(OiPr) $3(9.60 \mathrm{mg}, 0.03 \mathrm{mmol})$ was injected to the system for locking part living species. And then this operation will be repeated one time every 15 minutes in the following 45 minutes. 3 hours later, $t$-BuOK $(1000 \mu \mathrm{L}, 0.20 \mathrm{mmol})$ was ingected to the locked system, and after $30 \mathrm{~min}$, the mixture of DPE-SiH $(0.24 \mathrm{~g}, 1.00 \mathrm{mmol})$, St $(0.11 \mathrm{~g}, 1.00 \mathrm{mmol})$ and benzene $(10 \mathrm{w} / \mathrm{v} \%)$ was added for unlocking polymerization. 48 hours later, the polymerization was terminated with isopropanol. The product was precipitated with sufficient methanol and subsequently dissolved in an appropriate amount of toluene; this process was repeated twice to ensure that the residual 
monomers were completely removed.

The synthesis of polyisoprene with alkynyl (PIp-yne) functionalized was based on the previous reports ${ }^{6}$ and the characteristics for that were shown in FigureS2 and Figure S3.

After the backbones (BP-PS- $b$-PSD) and the branches (PIp-yne) were all prepared, the grafting-onto method was used here with an efficient hydrosilylation reaction. The backbone chains (BP-PS- $b$-PSD: $1.00 \mathrm{~g}$ ) and branched chains (PIp-yne: $1.20 \mathrm{~g}$ ) were firstly dissolved in toluene, and then keeping stirred, Karstedt's catalyst $(\mathrm{SiH} / \mathrm{Pt}=40 / 1)$ was added into the mixture. The reaction was allowed to react for $12 \mathrm{~h}$ at room temperature under an argon atmosphere, and the same above-mentioned method was used to precipitate the product. The block-graft copolymer was then separated from the residual branched chains of the feed using toluene and methanol. Finally, the product was dried in a vacuum oven to constant weight for further characterized.

\section{Additional Characteristics of the Polymers}
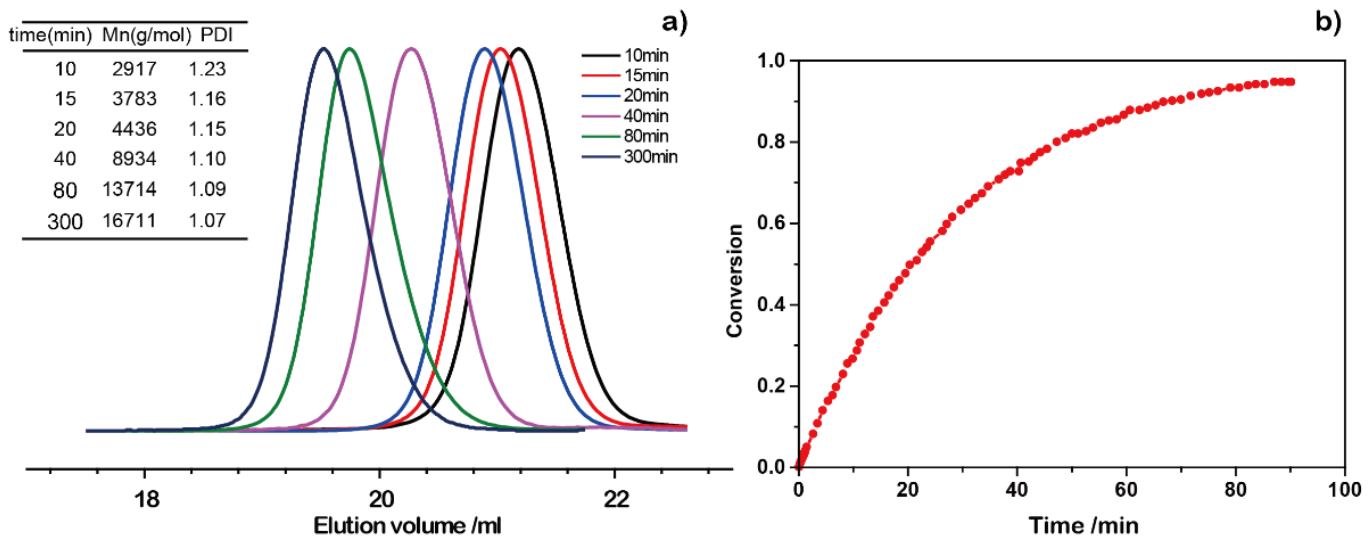

Figure S1. a) SEC curves for timely sampling for the living anionic homo-polymerization of styrene; b) the kinetic curves for the anionic homo-polymerization of styrene ${ }^{7}$.

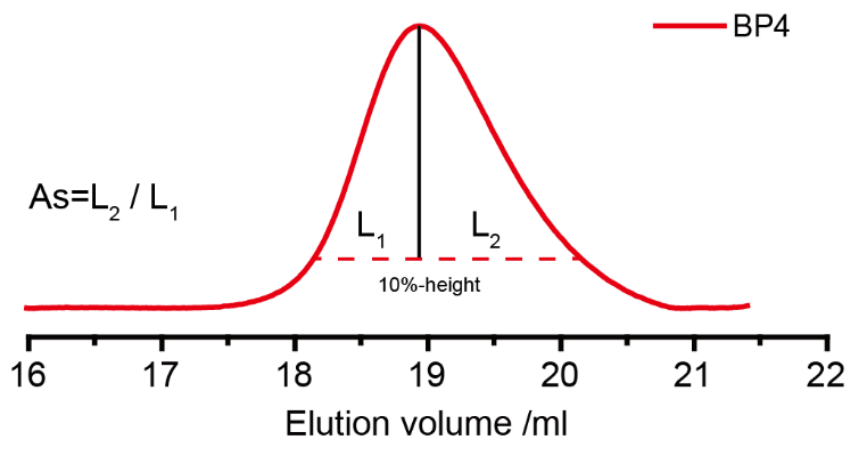


Figure S2. Calculation of the symmetric index As (It follows the methods in references ${ }^{8-9}$ )

a)

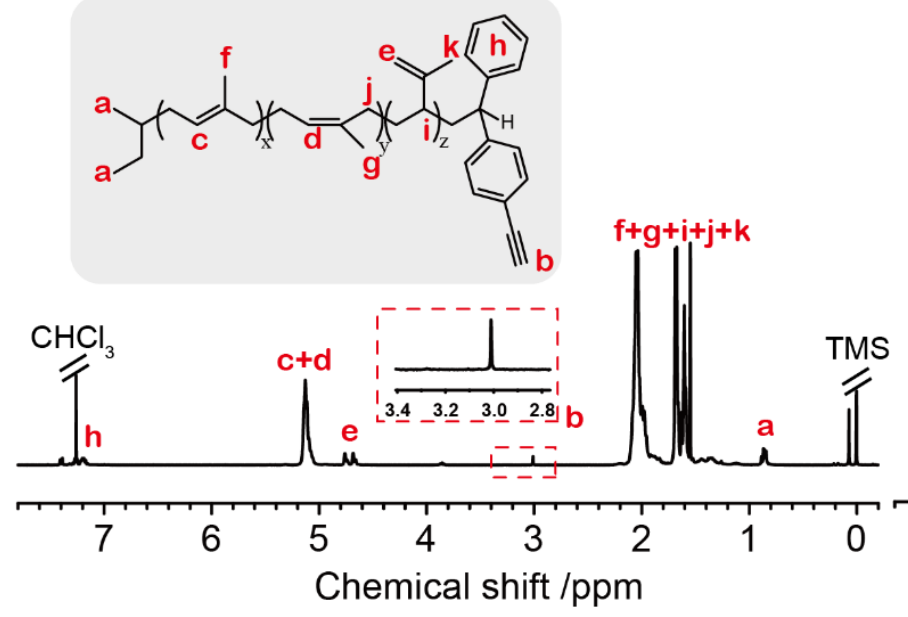

b)

$M n=6.1 \mathrm{~kg} / \mathrm{mol}$

$P D I=1.09$

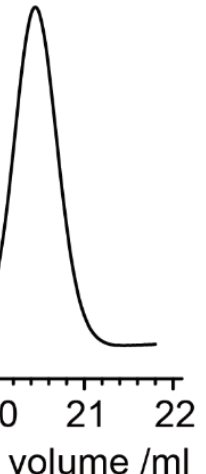

Figure S3. a) ${ }^{1} \mathrm{H}$ NMR spectrum and b) SEC curve of the alkynyl functionalized polyisoprene PIp-yne

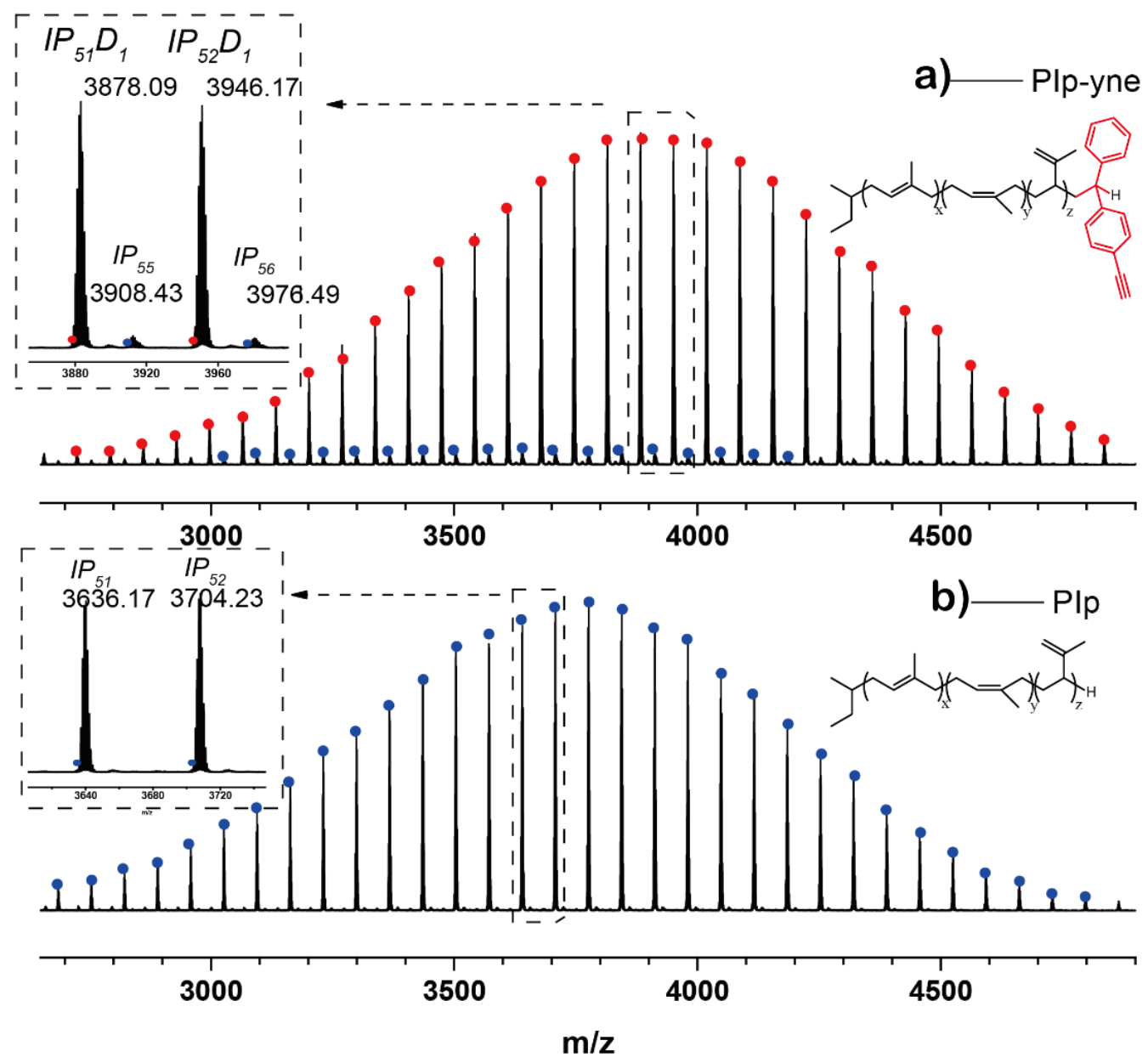

Figure S4. MALDI-TOF-MS of a) PIp-yne; b) PIp chains without alkynyl functionality 


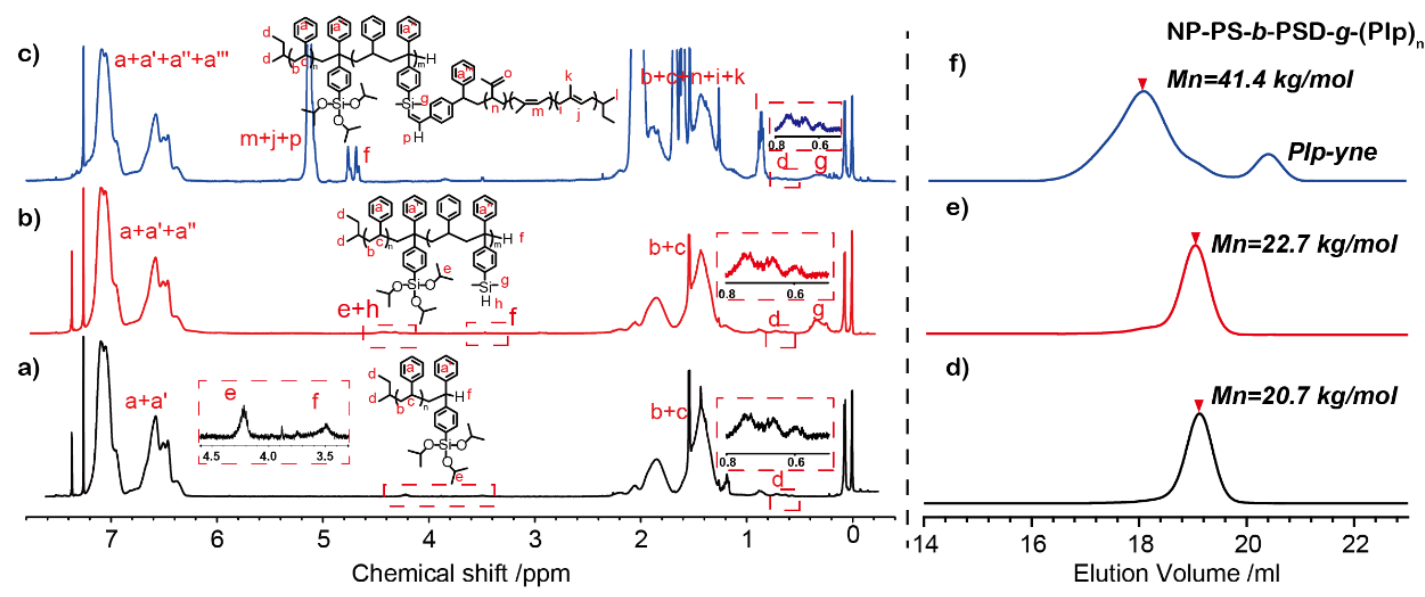

Figure S5. ${ }^{1} \mathrm{H}$ NMR spectra: a) Narrow-Peaks Polystyrene; b) NP backbone copolymers; c) block-graft copolymer and SEC curves of samples during the preparation of NP-PS- $b$-PSD- $g$-(PIp)n: d) Narrow-Peaks Polystyrene; e) NP backbone copolymers; f) block-graft copolymer
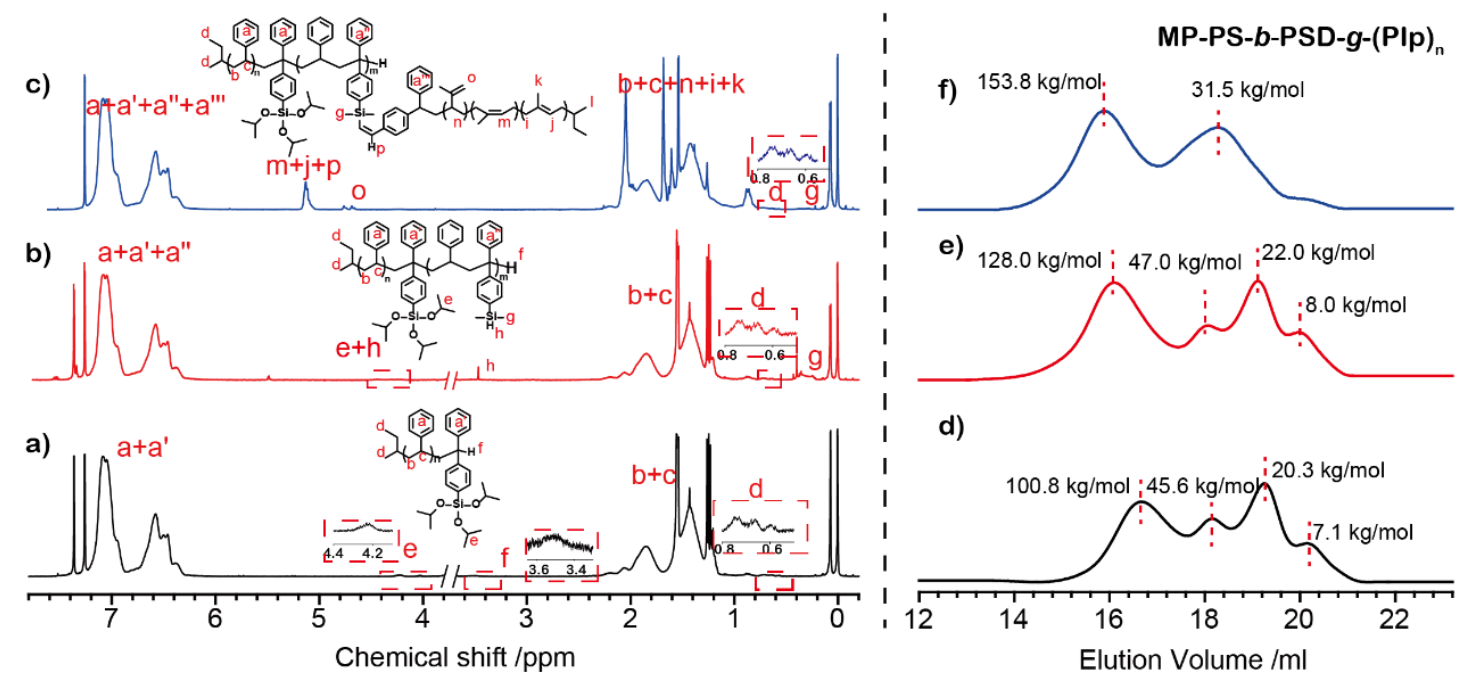

Figure S6. ${ }^{1} \mathrm{H}$ NMR spectra: a) Multi-Peaks Polystyrene; b) MP backbone copolymers; c) block-graft copolymer and SEC curves of samples during the preparation of MP-PS- $b$-PSD- $g$-(PIp)n: d) Multi-Peaks Polystyrene; e) MP backbone copolymers; f) block-graft copolymer

Figure $5 \mathrm{a}$ and $\mathrm{b}$ show the ${ }^{1} \mathrm{H}$ NMR spectra for the preparation of BP-PS and BP-PS-b-PSD. As depicted, the changes in $\delta=4.15-4.40 \mathrm{ppm}$ were the signal of proton of $\mathrm{SiH}$ group marked as $\mathrm{g}$. The signal difference around $\delta=3.5 \mathrm{ppm}$ is indicative of that the replacement between the end proton of DPE-Si(OiPr $)_{3}$ and a few end-protons of DPE-SiH and the new signals in $\delta=0.45-0.19 \mathrm{ppm}$ were the methyl-proton of DPE-SiH. Moreover, the number of $\mathrm{SiH}$ groups shown in Table 2 can also be calculated by the integral of these protons 
as the footnotes a) of Table 2 shows. Analogously, the ${ }^{1} \mathrm{H}$ NMR spectra for the synthesis of NP-PS-b-PSD and MP-PS- $b$-PSD also showed the same changes of the proton signal (see Figure S1 and S2).

For ${ }^{1} \mathrm{H}$ NMR spectra of the grafting reactions, as depicted, after the functionalized PIp grafted, a unique proton appeared marked as $\mathrm{n}$, but the chemical shift for that was superimposed to the protons of $=\mathrm{CH}$ - in the 1,4 addition of isoprene. However, as calculated, the Area $(\mathrm{n}+\mathrm{h}+\mathrm{j}) / \operatorname{Area}(\mathrm{f})$ for the final copolymer BP-PS- $b$ PSD-g-(PIp) in Figure 5c is 6.77, but the related signals for PIp was 6.44 (seeing Table S1). The increasing for that was consistent with the protons of $=\mathrm{CH}$ - generated by the hydrosilylation. Similarly, the ${ }^{1} \mathrm{H}$ NMR for MP-PS- $b$-PSD- $g-(P I p)_{\mathrm{n}}$ and NP-PS- $b$-PSD- $g-(\mathrm{PIp})_{\mathrm{n}}$ were also characterized shown in Figure S1 and S2, and the same results were detected in those spectra as BP-PS- $b$-PSD-g-(PIp)n.

Table S1. Results of the calculation in ${ }^{1} \mathrm{H}$ NMR spectrum for the unique proton after grafted

\begin{tabular}{|c|c|c|}
\hline Polymers & $\operatorname{Area}(n+h+j) / \operatorname{Area}(f)^{\mathrm{a})}$ & $H_{(1,4-\text { vinyl })} / H_{(3,4-\text { vinyl })}{ }^{\mathrm{b})}$ \\
\hline NP-PS- $b$-PSD- $g$-(PIp) $)_{\mathrm{n}}$ & 6.83 & - \\
\hline BP-PS- $b$-PSD- $g-(P I p)_{n}$ & 6.79 & - \\
\hline MP-PS- $b$-PSD- $g-(\mathrm{PIp})_{\mathrm{n}}$ & 6.77 & - \\
\hline PI & - & 6.44 \\
\hline
\end{tabular}

a) Area $(\mathrm{n}+\mathrm{h}+\mathrm{j})$ is the integral for the proton of $=C \boldsymbol{H}$ - in the block-graft copolymer, such as the peaks marked as $\mathrm{n}+\mathrm{h}+\mathrm{j}$ in Figure S6c. Area(f) refers to the proton of $=\mathrm{CH}_{2}$ in the block-graft copolymer, such as the peaks marked as $\mathrm{f}$ in Figure S6c; b) $H_{(1,4-\text { vinyl })}$ and $H_{(3,4-\text { vinyl })}$ were the proton of $=\boldsymbol{C H}$ - and $=\boldsymbol{C H}_{2}$ in the grafted chains PI-yne respectively, which were marked as $\mathrm{c}+\mathrm{d}$ and $\mathrm{e}$ in Figure $\mathrm{S} 3$.

Table S2. Data of DMA analysis

\begin{tabular}{lll}
\hline Polymers & Integral $(\tan \delta>0.3)$ & Temperature range $\left({ }^{\circ} \mathrm{C}\right)$ \\
\hline NP-PS- $b$-PSD- $g$-(PIp $)_{\mathrm{n}}$ & - & - \\
BP-PS- $b$-PSD- $g$-(PIp) & 0.56 & -60.0 to -34.1 \\
MP-PS- $b$-PSD- $g$-(PIp) & 7.69 & -60.0 to 49.8 \\
\hline
\end{tabular}




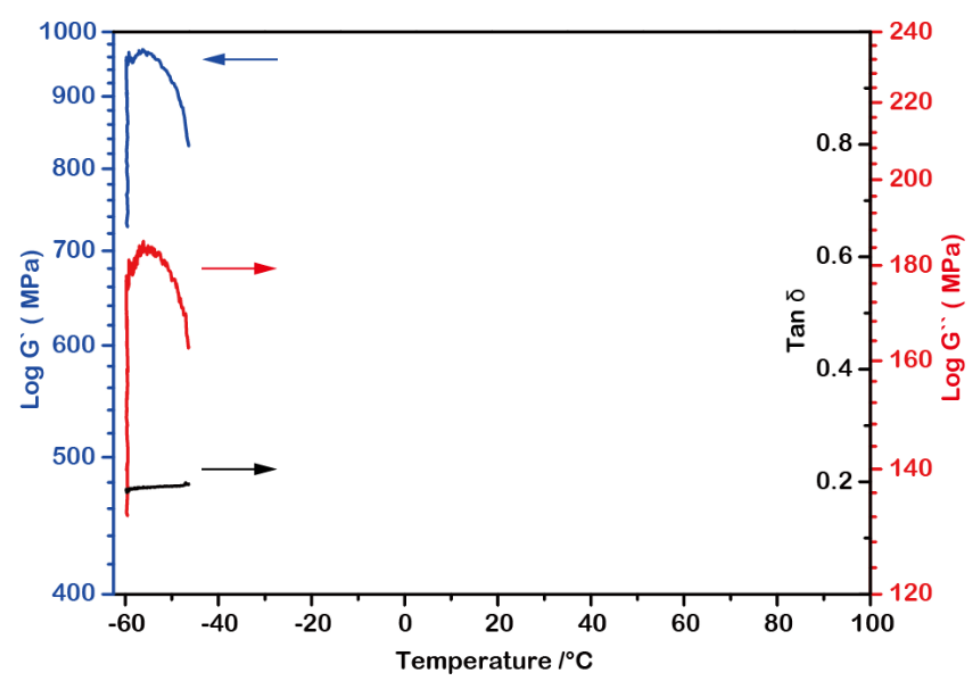

Figure S7. DMA data of NP-PS- $b$-PSD- $g-(P I p)_{\mathrm{n}}$

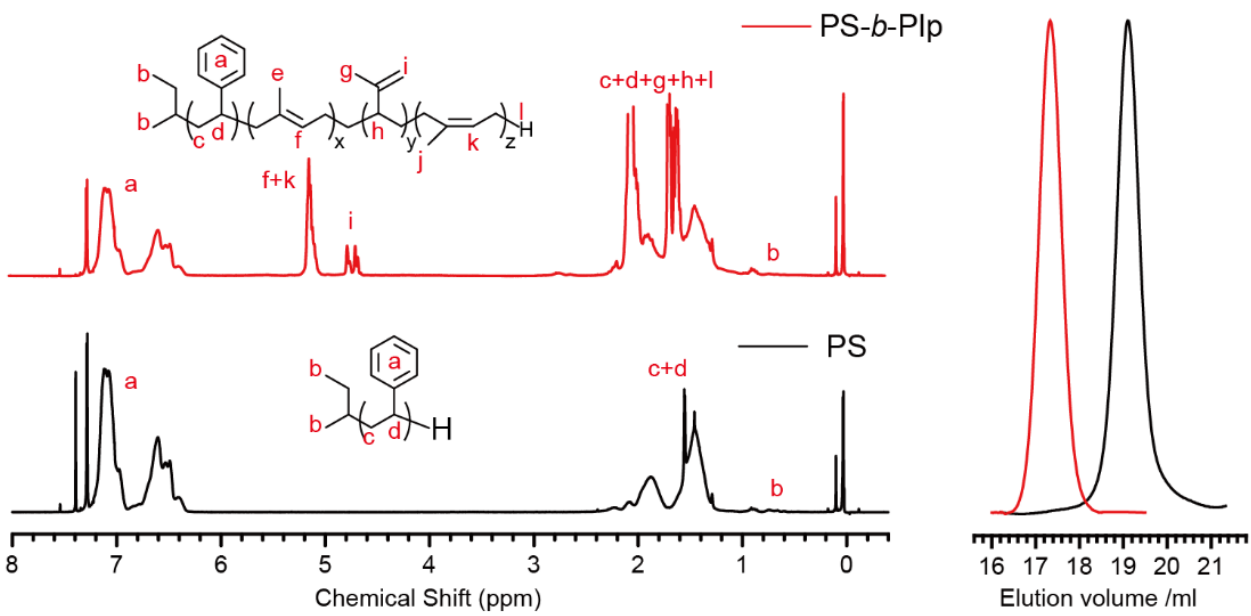

Figure S8. ${ }^{1} \mathrm{H}$ NMR spectrum and SEC curves of the block copolymer PS- $b$-PIp

\section{Reference}

1. Ma, H.; Han, L.; Li, Y., Sequence Determination and Regulation in the Living Anionic Copolymerization of Styrene and 1,1-Diphenylethylene (DPE) Derivatives. Macromolecular Chemistry and Physics 2017, 218, 1600420.

2. Zhang, Y.; Han, L.; Ma, H.; Yang, L.; Liu, P.; Shen, H.; Li, C.; Li, Y., The investigation on synthesis of periodic polymers with 1,1-diphenylethylene (DPE) derivatives via living anionic polymerization. Polymer 2019, 169, 95-105.

3. Li, C.; Leng, X.; Han, L.; Bai, H.; Yang, L.; Li, C.; Zhang, S.; Liu, P.; Ma, H., Unlocking features of locked-unlocked anionic polymerization. Polymer Chemistry 2020, 11 (48), 7696-7703.

4. Yang, L.; Ma, H.; Han, L.; Hao, X.; Liu, P.; Shen, H.; Li, Y., Synthesis of a sequence-controlled in-chain alkynyl/tertiary amino dual-functionalized terpolymer via living anionic polymerization. Polymer Chemistry 2017, 9, 108-120. 
5. Sang, W.; Ma, H.; Wang, Q.; Hao, X.; Zheng, Y.; Wang, Y.; Li, Y., Monomer sequence determination in the living anionic copolymerization of styrene and asymmetric bi-functionalized 1,1-diphenylethylene derivatives. Polymer Chemistry 2016, 7, 219-234.

6. Ma, Q.; Han, L.; Ma, H.; Liu, P.; Shen, H.; Yang, L.; Li, C.; Hao, X.; Li, Y., Investigation of the features of alternating copolymerization of 1,1-bis(4-dimethylsilylphenyl)ethylene and isoprene modified with additive. Polymer 2019, 184, 121907.

7. Quinebèche, S.; Navarro, C.; Gnanou, Y.; Fontanille, M., In situ mid-IR and UV-visible spectroscopies applied to the determination of kinetic parameters in the anionic copolymerization of styrene and isoprene. Polymer 2009, 50, 1351-1357.

8. Kottisch, V.; Gentekos, D. T.; Fors, B. P., "Shaping" the Future of Molecular Weight Distributions in Anionic Polymerization. ACS Macro Letters 2016, 5, 796-800.

9. Gentekos, D. T.; Dupuis, L. N.; Fors, B. P., Beyond Dispersity: Deterministic Control of Polymer Molecular Weight Distribution. Journal of the American Chemical Society 2016, 138, 1848-1851. 